\title{
A MODEL OF GLUCOCORTICOID RECEPTOR UNFOLDING AND STABILIZATION BY A HEAT SHOCK PROTEIN COMPLEX
}

\author{
William B. Pratt, * Lawrence C. Scherrer, Kevin A. Hutchison and \\ Friedrich C. DALMAN \\ The Department of Pharmacology, The University of Michigan Medical School, \\ Ann Arbor, MI 48109, U.S.A
}

\begin{abstract}
Summary-It has recently been reported that incubation of avian progesterone receptors, mouse glucocorticoid receptors, or the viral tyrosine kinase pp $60^{\text {src }}$ with rabbit reticulocyte lysate reconstitutes their association with the $90 \mathrm{kDa}$ heat shock protein, hsp90. The reassociation is thought to require unfolding of the steroid receptor or pp60 can bind. The unfoldase activity may be provided by hsp70, which is also present in the reconstituted receptor heterocomplex. In this paper we review evidence that hsp 70 and hsp90 are associated in cytosolic heterocomplexes that contain a limited number of other proteins. From an analysis of known receptor-hsp interactions and a predicted direct interaction between hsp90 and hsp70 we have developed an admittedly very speculative model of glucocorticoid receptor unfolding and stabilization. One important feature of the model is that the receptor becomes attached to a heat shock protein heterocomplex rather than undergoing independent unfolding and stabilization events. The model requires that hsp 70 and hsp 90 bind directly to the receptor at independent sites. Importantly, the model accommodates the stoichiometry of $2 \mathrm{hsp} 90$ per 1 molecule of receptor that has been assayed in the untransformed GR heterocomplex in cytosols prepared from hormone-free cells.
\end{abstract}

\section{INTRODUCTION}

Upon lysis of hormone-free cells in buffers of low ionic strength, glucocorticoid receptors (GR) are recovered in the cytosolic fraction as $\sim 9 \mathrm{~S}$ heteromeric complexes that do not have DNAbinding activity (see Ref. [1] for review). The "core" unit of this untransformed receptor consists of a dimer of hsp90 [2,3], which is bound to the receptor via protein-protein interactions in the hormone-binding domain $[4,5]$. When glucocorticoids bind to the cytosolic GR, they promote a temperature-dependent dissociation of the receptor from hsp 90 and the simultaneous transformation of the receptor from a nonDNA-binding form to a DNA-binding form [6-8]. Because the hormone also promotes dissociation of the GR from hsp 90 in the intact cell [9] and because association of the GR with hsp90 correlates with the hormone-inducible versus constitutive activity of receptor mutants [4], our

Proceedings of the 10th International Symposium of the Journal of Steroid Biochemistry and Molecular Biology, Recent Advances in Steroid Biochemistry and Molecular Biology, Paris, France, 26-29 May 1991.

*To whom correspondence should be addressed. laboratory has studied the steroid-promoted dissociation of the GR from hsp90 in cytosol as a model system for what is arguably the initial event in glucocorticoid hormone action. Cytosolic progesterone receptors undergo a similar steroid-promoted dissociation from hsp 90 $[10,11]$.

Despite the fact that the GR is tightly bound to hsp90 when it is isolated from hormone-free cells, purified hsp90-free GR does not reassociate with purified hsp90. It seems that after hsp90 has dissociated from the receptor, the conformation of the receptor is changed such that reassociation cannot occur. Thus, the receptor-hsp90 complex does not reflect a simple equilibrium binding. This led our laboratory [12] and that of Denis and Gustafsson [13] to ask whether GR that was translated in a reticulocyte lysate would associate with hsp90. It was found that the newly translated GR binds to hsp90 at or near the termination of receptor translation [12], forming a complex that can be converted to the DNA-binding form after binding with steroid and heat treatment [13]. Smith et al. [14] then demonstrated that rabbit reticulocyte lysate carries out the temperature-dependent association of rabbit hsp 90 with immunopurified avian 
progesterone receptor, and we subsequently performed a similar reconstitution of the murine GR-hsp90 complex [15]. The reticulocyte lysate apparently contains a complete system for receptor unfolding and for subsequent stabilization of the unfolded form with hsp90. In the case of the GR, it has been shown that reassociation of the receptor with hsp 90 is accompanied by functional reconstitution of the untransformed state of the receptor [15]. We have recently shown that the rabbit reticulocyte lysate also reconstitutes the complex of $\mathrm{pp} 60^{\mathrm{scc}}$ with both hsp90 and p50 [16], suggesting that the reconstitution of steroid receptor-hsp90 complexes may represent a more general protein unfolding activity of the reticulocyte lysate.

STRUCTURAL AND FUNCTIONAL RECONSTITUTION OF THE GR-hsp90 COMPLEX

In several studies $[6,8]$ we have shown that the presence of hsp90 is associated with the non-DNA-binding state of the glucocorticoid receptor in L cell cytosol, and recently we have shown that there is a direct correlation between the presence of hsp90 and high affinity steroid binding activity $[17,18]$. Indeed, under no conditions have we been able to find an hsp90free glucocorticoid receptor that binds steroid, suggesting that hsp90 must be bound to the receptor for the hormone binding domain to be in a high affinity glucocorticoid binding conformation. This differs from most of the other well studied steroid receptors (e.g. estrogen and progesterone receptors) which bind ligand in their hsp90-free state.

This requirement of hsp90 for steroid binding seems to pertain to rat GR that is translated in vitro as well. We chose two in vitro systems for translating the GR, rabbit reticulocyte lysate and wheat germ lysate [12]. Rabbit reticulocyte lysate contains approximately $2 \mu \mathrm{M}$ hsp 90 , whereas we have been unable to establish the existence of an analogous protein in wheat germ lysate by immunoblotting with anti-hsp 90 antibodies that react with a wide spectrum of hsp90s from the mammalian kingdom. We found that GR translated in the hsp90-containing translation system (reticulocyte) has a normal glucocorticoid binding activity and must be

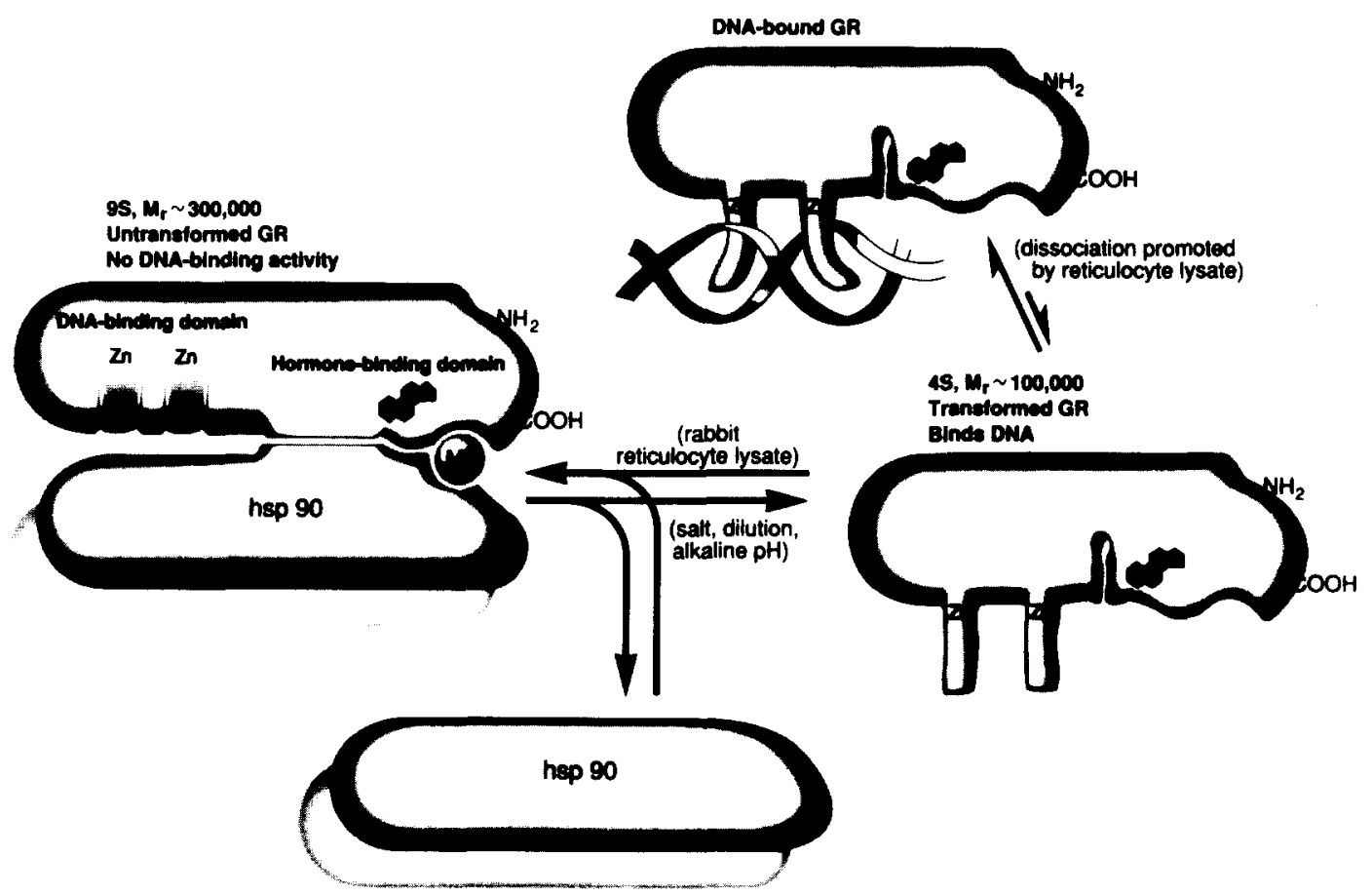

Fig. 1. Model of the GR-hsp90 interaction. Hsp90 binds to the hormone binding domain of the receptor to form a complex without DNA binding activity, which is stabilized by molybdate (indicated by the small globe with the $\mathbf{M}^{=}$). Dissociation of the complex is accompanied by transformation to the DNA binding state (indicated by the receptor with the exposed "zinc fingers" on the right). Unliganded, monomeric receptor that has been either immunoadsorbed to protein A-Sepharose or bound to DNA-cellulose can be reassociated with hsp90 in a temperature-dependent reaction directed by rabbit reticulocyte lysate. Reassociation with hsp90 reconstitutes the non-DNA-binding, untransformed receptor. From Scherrer et al. [15]. 
transformed before it will bind to DNAcellulose. In contrast, GR synthesized in the plant system without hsp90 (at least without immunodetectible hsp90) does not have a high affinity ligand binding conformation and it is translated in the DNA-binding form $[12,19]$. Thus, the untransformed, cytosolic GR-hsp90 complex differs from the hsp90-free GR in both its DNA-binding and steroid-binding activities.

Toft and his colleagues [14] made a major advance in the study of receptor-hsp90 complexes when they demonstrated that incubation of immunopurified chick progesterone receptor with rabbit reticulocyte lysate reconstituted a receptor heat-shock protein complex. In this case, the chicken progesterone receptor became associated in a temperature-dependent manner with rabbit hsp90 and hsp 70 . The unliganded receptor reconstituted with heat shock proteins whereas the steroid-bound receptor did not, consistent with the role of the ligand in promoting dissociation. We have immunoadsorbed the salttransformed, unliganded, hsp90-free GR from $L$ cell cytosol and shown that incubation of the immune pellet with reticulocyte lysate reconstitutes a GR-hsp90-hsp70 complex [15].

A model of the dissociation and reconstitution of the GR heterocomplex is presented in Fig. 1. In the case of the GR, we were able to ask whether the GR-heat shock protein complex was functionally as well as structurally reconstituted. That is, we showed that the reconstitution of the GR-hsp90 complex was accompanied by conversion of the receptor from the DNAbinding to a non-DNA-binding state and from a form that does not bind steroid to a form that binds steroid with high affinity [15]. If unliganded receptors are dissociated from hsp90 and bound to DNA-cellulose, subsequent incubation with reticulocyte lysate causes their temperature-dependent release from DNA. The GR that is released from DNA has been reconstituted into a GR-hsp90 complex and it has also been converted to a high affinity steroid binding form. Thus, from both a structural and a functional point of view, the experiments with reticulocyte lysate represent the first demonstration of the reversal of steroid receptor transformation.

Several years before steroid receptors were first shown to be associated with hsp 90 , it was shown that $\mathrm{pp} 60^{\mathrm{v}-\mathrm{src}}$ associates with hsp90 and a $50-\mathrm{kDa}$ protein of unknown function, p50 (see Ref. [20] for review). The pp60 $0^{v-s r c}$ protein encoded in Rous sarcoma virus is a membraneassociated tyrosine kinase that induces oncogenic transformation of cells. As soon as it is translated, pp60 ${ }^{\text {s-src }}$ associates with hsp 90 and p50 and it remains transiently associated with these proteins until it localizes to cell membranes where pp $60^{v-s r c}$ dissociates from the complex. It is thought that hsp90 and p50 are involved in translocation of the cytoplasmic pp60 $60^{\text {v-src }}$ to cell membranes.

As with the steroid receptors, it has not been possible to reconstitute the pp60 ${ }^{\text {v-src }}-\mathrm{hsp} 90$ complex using purified components. We have shown [16], however, that incubation of immunopurified $\mathrm{pp} 60^{v-s r c}$ with rabbit reticulocyte lysate results in reconstitution of the complex between the tyrosine kinase and both hsp90 and p50. In addition, as previously shown with progesterone and glucocorticoid receptors, there is also immune-specific association of the $\mathrm{pp} 60^{\mathrm{v}-\mathrm{src}}$ with hsp70.

\section{EVIDENCE THAT hsp90 AND hsp70 ACT AS PART OF A MULTIPROTEIN COMPLEX}

An important concept arising from a recent series of publications is that several heat shock proteins interact both with themselves and with some other proteins in cytosol to form a multiprotein complex. The general approach has been to immunoadsorb a steroid receptor or a heat shock protein with a monoclonal antibody specific for that protein and then to determine what other proteins are coimmunoadsorbed with

Table 1. Common protein complexes indicated by coimmunoprecipitation of proteins from cytosol

\begin{tabular}{|c|c|c|c|c|c|}
\hline Laboratory & $\begin{array}{l}\text { Toft } \\
{[10,11]}\end{array}$ & $\begin{array}{l}\text { Pratt } \\
{[3,23,27]}\end{array}$ & $\begin{array}{l}\text { Perdew } \\
{[30,31]}\end{array}$ & $\begin{array}{l}\text { Pratt } \\
{[27]}\end{array}$ & $\begin{array}{l}\text { Brugge } \\
{[20]}\end{array}$ \\
\hline $\begin{array}{l}\text { Immunoadsorbed protein } \\
\text { Associated protein }\end{array}$ & $\begin{array}{l}\text { PR } \\
\text { hsp90 } \\
\text { hsp70 } \\
\text { p54 (?hsp56) } \\
\text { p50 } \\
\text { p23 }\end{array}$ & $\begin{array}{l}\text { GR } \\
\text { hsp90 } \\
\text { hsp70 } \\
\text { hsp56 } \\
\text { p23 }\end{array}$ & $\begin{array}{l}\text { hsp90 } \\
\text { hsp90 } \\
\text { hsp70 } \\
\text { hsp 56 } \\
\text { p50 }\end{array}$ & $\begin{array}{l}\text { hsp56 } \\
\text { hsp90 } \\
\text { hsp70 } \\
\text { hsp56 } \\
\text { p23 }\end{array}$ & $\begin{array}{l}\text { pp60 } \\
\text { hsp90 } \\
\text { p50 }\end{array}$ \\
\hline
\end{tabular}


it (see Ref. [21] for review). The proteins identified in untransformed progesterone and glucocorticoid receptor complexes are summarized in Table 1.

If cytosolic glucocorticoid receptors are immunoadsorbed and washed rigorously, it can be shown that two molecules of hsp90 are bound per molecule of GR [2,3], consistent with the known behavior of hsp90 as a dimer in solution [22]. When unliganded glucocorticoid receptors are isolated from cytosol under the gentlest conditions designed to maintain as much of the structure of the native receptor as possible, higher order complexes can be readily detected [3]. The higher order GR complexes in L cell cytosol contain more than one dimer of hsp90, as well as other proteins of apparent $M_{r} 23,000$ and 55,000 [3]. Although the untransformed GR heterocomplex in L cell cytosol does not contain hsp 70, mouse GR expressed in $\mathrm{CHO}$ cells is clearly associated with hsp70 as well as hsp90 [23].

Hsp70 was first demonstrated to be a component of the untransformed progesterone receptor in cytosol prepared from chick oviduct. In careful experiments, Kost et al. [10] and Smith et al. [11] have shown that rapid gentle immunoadsorption of avian progesterone receptors results in the immunospecific isolation of four proteins of approximately 70,54, 50 and $23 \mathrm{kDa}$ in addition to hsp 90 . The $70-\mathrm{kDa}$ protein has been identified as the constitutive form of hsp70. Unlike hsp90 and the other proteins, hsp 70 does not dissociate when the progesterone receptor is transformed [11]. It has also been shown that hsp70 does not dissociate from the receptor when the mouse GR in CHO cell cytosol is transformed [23].

Several years agao, Nakao et al. [24] developed a monoclonal antibody against the untransformed rabbit progesterone receptor heterocomplex. This ECl antibody reacts with a $59-\mathrm{kDa}$ rabbit protein that is contained in 9S untransformed progestin, estrogen, androgen and glucocorticoid receptor complexes [25]. Cross-linking experiments suggest that this protein is bound to hsp90 and is not directly associated with the receptors themselves [26]. In human lymphocyte cytosol, this protein is a $56-\mathrm{kDa}$ protein with several isomorphs [27]. Recently, Sanchez [28] has shown that this p56 is synthesized at an increased rate when human lymphocytes are submitted to heat shock conditions, leading to its new designation as hsp56. Because the ECl antibody does not react with chicken proteins, it is not yet known if the p54 identified in the avian receptor complex is hsp56. It seems likely this is the case, however, as hsp 56 is an established component of the heteromeric rabbit progesterone complex $[25,26]$. Because hsp56 has been identified in both rabbit $[25,26]$ and human [27] heteromeric GR complexes, it is also likely that hsp56 is the $M_{r} 55,000$ protein with several isomorphs that was found to copurify with the mouse L cell GR-hsp90 complex [3].

In an important series of experiments, Perdew and his colleagues have used the $8 \mathrm{D} 3$ monoclonal IgM antibody [29] to immunoadsorb hsp90 from Hepa 1 cell cytosol and identify hsp90-associated proteins. Three major proteins that immunoadsorb with hsp90 are hsp70, hsp56 and a protein with $\mathrm{M}_{\mathrm{r}} 50,000$ [30]. This $50-\mathrm{kDa}$ protein has now been identified as the same $50-\mathrm{kDa}$ phosphoprotein (p50) that is present in the pp60 $0^{v-s r c}-$ hsp90 complex [31]. In a similar approach, we have shown that immunoadsorption of hsp56 with the EC1 monoclonal antibody results in coadsorption of large amounts of hsp90 and hsp70, as well as variable amounts of a $23-\mathrm{kDa}$ protein. The relationship between the $23-\mathrm{kDa}$ proteins found in avian progesterone receptor and mouse glucocorticoid receptor heterocomplexes is as yet unknown, as is the relationship between these two proteins and the $\mathrm{p} 23$ recovered with the hsp56 complex.

The unifying concept that derives from all of these studies is that hsp90, hsp70 and some other proteins, like hsp 56 and p50, somehow interact with each other as components of a heteromolecular complex that is present in hypotonic cytosols. Those steroid receptors that are recovered from hormone-free cells in the cytosolic fraction after hypotonic lysis (glucocorticoid, mineralocorticoid, dioxin, progestin, estrogen and androgen receptors) all remain in an inactive "docking" complex that is located either in the cytoplasm or in the cell nucleus until binding of hormone triggers the progression of the receptor to high affinity nuclear binding sites where the primary events involved in transcriptional activation occur. We have previously developed the argument that the steroid receptor heterocomplex is derived from this "docking" structure, and that the 9S, untransformed state of the cytosolic steroid receptors represents a "core" unit of the heterocomplex [3,27, see Ref. 21 for a review]. This proposal is supported by the observation that the thyroid hormone receptor and the retinoic 
acid receptor do not form stable complexes with hsp90 $[19,32]$. Both of these receptors become very tightly associated with the nucleus in hormone-free cells without prior retention in a docking complex.

The docking complex may have evolved from a general role of hsp70 and hsp90 in protein folding and stabilization. Members of the hsp70 family have been shown to interact co-translationally with a variety of proteins [33] and there is considerable evidence that hsp70 (and its prokaryotic homolog the dna K gene product [34]) promote protein unfolding and maintenance of the "unfolded" state for passage of proteins across biological membranes (see Ref. [35] for review). It seems quite possible that hsp90 also associates transiently with a variety of proteins when they are translated in the cell. This may very well be the case with thyroid hormone and retinoic acid receptors, but the affinity of the complex that is formed may not be high enough to permit detection with our methods.

\section{A MODEL OF HEAT SHOCK PROTEIN- MEDIATED UNFOLDING AND STABILIZATION OF THE GLUCOCORTICOID RECEPTOR}

Until 1990, steroid receptor-hsp90 interactions were considered only from a bimolecular viewpoint. It now seems likely that multiple proteins will be found to participate in the reconstitution of steroid receptor-hsp90 complexes in reticulocyte lysate. Indeed, as discussed above, it is reasonable to consider the possibility that the receptor becomes attached to a multiprotein complex. The common components of the progesterone receptor, glucocorticoid receptor and $\mathrm{pp} 60^{\mathrm{scc}}$ heterocomplexes that are formed in reticulocyte lysate are hsp70 and hsp90, and others may exist. Smith et al. [14] have identified 23-kDa and $60-\mathrm{kDa}$ proteins (by Coomassie blue staining) in the lysate-reconstituted chicken progesterone complex. It is not yet known if these proteins are present in the reconstituted GR or pp60 $0^{\mathrm{v}-\mathrm{src}}$ complexes. The fact that $\mathrm{p} 50$ is clearly present in reconstituted pp60 $0^{v-s r c}$ complexes but has not been identified in reconstituted glucocorticoid [16] or progesterone [14] receptor complexes may suggest that the final heterocomplex that is formed is not the same in all cases (although simple stoichiometric considerations and methodological difficulties inherent to the study of weak protein-protein interactions could yield the apparent differences in heterocomplex composition observed to date).

At present, nothing is known about the mechanism of steroid receptor unfolding or reconstitution of receptors into the heterocomplex with heat shock proteins. One can, however, construct a model of the process based upon both established protein-protein interactions and some protein-protein interactions that would be predicted from the information discussed above. Figure 2 presents an unfolding model that is based on the known unfoldase activity of hsp70 and assumes that hsp90 passively stabilizes the unfolded conformation of the receptor.

Because hsp90 behaves as a dimer, both in solution [22] and when it is bound to the GR $[2,3]$, it is reasonable to assume there is a dimerization site (solid line in Fig. 2). Although it has not been proven by cross-linking that hsp90 is bound to hsp70 via direct proteinprotein interactions, the fact that abundant amounts of each protein have been identified in heterocomplexes obtained (independent of the

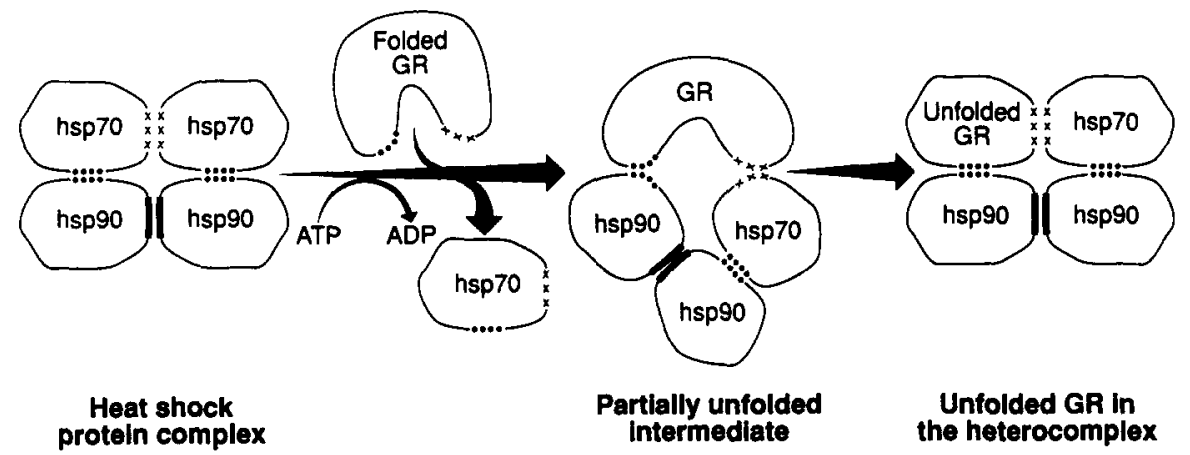

Fig. 2. A model of heat shock protein-mediated unfolding and stabilization of the glucocorticoid receptor. Sites of protein-protein interaction are indicated by the following symbols: bold lines, hsp 90 dimerization site; $\cdots$, , site of high affinity interaction of GR with hsp90 or low affinity interaction of hsp70 with hsp90; $\times \times \times$, site of high affinity interaction of GR with hsp70. Note that the GR and hsp70 may interact with hsp90 at completely different sites. See text for details of the model. 
presence of steroid receptors) by coimmunoadsorption with heat shock protein antibodies $[27,30]$ makes this suggestion reasonable. It is known that hsp70 purifies as a monomer, but if it binds to a dimer of hsp90 in cytosol, then two molecules of hsp70 might be present in the predicted protein unfolding complex.

In considering the binding of the steroid receptor to such a protein unfolding complex, two known protein-protein interactions must be considered. First, there must be a direct protein-protein interaction between hsp90 and the hormone binding domain of the receptor. The hormone binding domain of the GR is clearly required for heterocomplex formation $[4,5,36-38]$ and a similar requirement has been shown for the progesterone receptor [39]. The results of cross-linking studies with both receptors are consistent with a direct protein-protein interaction between the receptor and hsp 90 $[40,41]$. Second, there must be a direct proteinprotein interaction between hsp70 and the receptor. This is inferred from the observation that $\mathrm{hsp} 70$ remains associated with cytosolic progesterone and glucocorticoid receptors when they are transformed and hsp90 dissociates $[11,23]$. There is some evidence that the steroidbinding domain of the progesterone receptor is needed for hsp70 binding [14]. The receptorhsp70 complex survives vigorous washing (including with salt) and is dissociated in an ATP-dependent manner [10].

Of the interactions shown in the model, the binding of hsp90 to itself, the binding of hsp90 to the receptor, and the binding of hsp70 to the receptor are all of high affinity. The interaction between hsp 70 and hsp90 is much weaker. Thus, one mechanism of entry of the folded GR into the complex might be by displacement of an hsp70. The site of GR interaction on hsp90 is drawn in Fig. 2 as the same site where hsp70 binds to hsp90, but these sites could be different. We find that the GR reconstitution process is both temperature-dependent and enhanced by the presence of an ATP generating system. Thus, we have indicated a requirement for ATP, although the step (or steps) in the process that requires ATP hydrolysis is unknown. It is assumed in the model that there must be two different contact sites between the folded GR and the hsp complex. This is consistent with hsp90 and hsp70 each having a different site of contact within the steroid binding domain. Subsequent to unfolding, hsp70 may be able to exit the complex, leaving the hsp90-stabilized (but hsp70-free) form of the GR that is seen in L cell cytosol, or it may remain bound to the heterocomplex as is seen when the same mouse receptor is expressed in $\mathrm{CHO}$ cells [27]. In either case, the GR is competent to bind steroid and it is repressed with respect to its DNA-binding activity [27]. Importantly, this model accommodates the stoichiometry of 2 hsp 90 per molecule of GR that has been assayed in the untransformed receptor core complexes in cytosols prepared from hormone-free cells. Although this model is both highly speculative and undoubtedly incomplete, it contains elements that can be tested if the reconstitution system in the reticulocyte lysate can be further resolved.

Acknowledgements - These investigations were supported by NIH Grants DK31573 and CA28010 from the National Cancer Institute.

\section{REFERENCES}

1. Pratt W. B.: Transformation of glucocorticoid and progesterone receptors to the DNA-binding state. J. Cell. Biochem. 35 (1987) 51-58.

2. Mendel D. B. and Orti E.: Isoform composition and stoichiometry of the $90-\mathrm{kDa}$ heat shock protein associated with glucocorticoid receptors. J. Biol. Chem. 263 (1988) 6695-6702.

3. Bresnick E. H., Dalman F. C. and Pratt W. B.: Direct stoichiometric evidence that the untransformed $M_{r}$ $300,000,9 \mathrm{~S}$, glucocorticoid receptor is a core unit derived from a larger heteromeric complex. Biochemistry 29 (1990) 520-527.

4. Pratt W. B., Jolly D. J., Pratt D. V., Hollenberg S. M., Giguere V., Cadepond F. M., Schweizer-Groyer G., Catelli M. G., Evans R. M. and Baulieu E. E.: A region in the steroid binding domain determines formation of the non-DNA-binding, 9S glucocorticoid receptor complex. J. Biol. Chem. 263 (1988) 267-273.

5. Denis M., Gustafsson J. A. and Wikstrom A. C.: Colocalization of the $M_{r} 90,000$ heat shock protein with the steroid binding domain of the glucocorticoid receptor J. Biol. Chem. 263 (1988) 18520-18523.

6. Sanchez E. R., Meshinchi S., Tienrungroj W., Schlesinger M. J., Toft D. O. and Pratt W. B.: Relationship of the 90-kDa heat shock protein to the untransformed and transformed states of the $\mathrm{L}$ cell glucocorticoid receptor. J. Biol. Chem. 262 (1987) 6986-6991.

7. Denis M., Poellinger L., Wikstrom A. C. and Gustafsson J. A.: Requirement of hormone for thermal conversion of the glucocorticoid receptor to a DNA-binding state. Nature, Lond. 333 (1988) 686-688.

8. Meshinchi S., Sanchez E. R., Martell K. J. and Pratt W. B.: Elimination and reconstitution of the requirement for hormone in promoting temperature-dependent transformation of cytosolic glucocorticoid receptors to the DNA-binding state. J. Biol. Chem. 265 (1990) 4863-4870.

9. Mendel D. B., Bodwell J. E., Gametchu B., Harrison R. W. and Munck A.: Molybdate-stabilized nonactivated glucocorticoid-receptor complexes contain a 90-kDa nonsteroid-binding phosphoprotein that is lost on activation J. Biol. Chem. 261 (1986) 3758-3763.

10. Kost S. L., Smith D., Sullivan W., Welch W. J. and Toft D. O.: Binding of heat shock proteins to the 
avian progesterone receptor. Molec. Cell. Biol. 9 (1989) 3829-3838.

11. Smith D. F., Faber L. E. and Toft D. O.: Purification of unactivated progesterone receptor and identification of novel receptor-associated proteins. J. Biol. Chem. 265 (1990) 3996-4003.

12. Dalman F. C., Bresnick E. H., Patel P. D., Perdew G. H., Watson S. J. and Pratt W. B.: Direct evidence that the glucocorticoid receptor binds to hsp90 at or near the termination of receptor translation in vitro. J. Biol. Chem. 264 (1989) 19815-19821.

13. Denis $M$. and Gustafsson J. A.: Translation of a glucocorticoid receptor $\mathrm{mRNA}$ in vitro yields a nonactivated protein. J. Biol. Chem. 264 (1989) 6005-6008.

14. Smith D. F., Schowalter D. B., Kost S. L. and Toft D. $O$.: Reconstitution of progesterone receptor with heat shock proteins. Molec. Endocr. 4 (1990) 1704-1711.

15. Scherrer L. C., Dalman F. C., Massa E., Meshinchi S. and Pratt W. B.: Structural and functional reconstitution of the glucocorticoid receptor-hsp90 complex. J. Biol. Chem. 265 (1990) 21397-21400.

16. Hutchison K. A., Brott B. K., De Leon J. H., Perdew G. H., Jove R. and Pratt W. B.: Reconstitution of the multiprotein complex of pp60 $60^{\text {scc }}, \mathrm{hsp} 90$ and $\mathrm{p} 50$ in a cell-free system. J. Biol. Chem. In press.

17. Bresnick E. H., Sanchez E. R. and Pratt W. B.: Relationship between glucocorticoid receptor steroid binding capacity and association of the $M_{z} 90,000$ heat shock protein with the unliganded receptor. J. Steroid. Biochem. 30 (1988) 267-269.

18. Bresnick E. H., Dalman F. C., Sanchez E. R. and Pratt W. B.: Evidence that the $90-\mathrm{kDa}$ heat shock protein is necessary for the steroid binding conformation of the $\mathrm{L}$ cell glucocorticoid receptor. J. Biol. Chem. 264 (1989) 4992-4997.

19. Dalman F. C., Koenig R. J., Perdew G. H., Massa E. and Pratt W. B.: In contrast to the glucocorticoid receptor, the thyroid hormone receptor is translated in the DNA binding state and is not associated with hsp 90 . J. Biol. Chem. 265 (1990) 3615-3618.

20. Brugge J. S.: Interaction of the Rous sarcoma virus protein pp60 $60^{\text {sc }}$ with the cellular proteins pp50 and pp90. Curr. Top. Microbiol. Immun. 123 (1986) 1-22.

21. Pratt W. B.: Interaction of hsp 90 with steroid receptors: organizing some diverse observations and promoting the newest concepts. Molec. Cell. Endocr. 74 (1990) C69-C76.

22. Denis M., Wikstrom A. C. and Gustafsson J. A.: The molybdate-stabilized nonactivated glucocorticoid receptor contains a dimer of $\mathrm{M}_{\mathrm{r}} \mathbf{9 0 , 0 0 0}$ nonhormone binding protein. J. Biol. Chem. 262 (1987) 11803-11806.

23. Sanchez E. R., Hirst M., Scherrer L. C., Tang H. Y., Welsh M. J., Harmon J. M., Simons S. S., Ringold G. M. and Pratt W. B.: Hormone-free glucocorticoid receptors overexpressed in Chinese hamster ovary cells are localized to the nucleus and are associated with both hsp70 and hsp90. J. Biol. Chem. 265 (1990) 20123-20130.

24. Nakao K., Myers J. E. and Faber L. E.: Development of a monoclonal antibody to the rabbit $8.5 \mathrm{~S}$ uterine progestin receptor. Can. J. Biochem. Cell. Biol. 63 (1985) 33-40.

25. Tai P. K., Maeda Y., Nakao K., Wakim N. G., Duhring J. L. and Faber L. E.: A 59-kilodalton protein associated with progestin, estrogen, androgen and glucocorticoid receptors. Biochemistry 25 (1986) 5269-5275.

26. Renoir J. M., Radanyi C., Faber L. E. and Baulieu E. E.: The non-DNA-binding heteroligomeric form of mammalian steroid hormone receptors contains a hsp90-bound 59-kilodalton protein. J. Biol. Chem. 265 (1990) 10740-10745.

27. Sanchez E. R., Faber L. E., Henzel W. J. and Pratt W. B.: The 56-59-kilodalton protein identified in untransformed steroid receptor complexes is a unique protein that exists in cytosol in a complex with both the 70- and 90-kilodalton heat shock proteins. Biochemistry 29 (1990) 5145-5152.

28. Sanchez E. R.: Hsp56: A novel heat shock protein associated with untransformed steroid receptor complexes. J. Biol. Chem. 265 (1990) 22067-22070.

29. Perdew G. H.: Association of the $A$ h receptor with the 90-kDa heat shock protein. J. Biol. Chem. 263 (1988) 13802-13805.

30. Perdew G. H. and Whitelaw M. L.: Evidence that the 90-kDa heat shock protein (hsp90) exists in cytosol in heteromeric complexes containing hsp70 and three other proteins with $M_{r}$ of $63,000,56,000$, and 50,000 . J. Biol. Chem. 266 (1991) 6708-6713.

31. Whitelaw M. L., Hutchison K. and Perdew G. H.: A $50 \mathrm{kDa}$ cytosolic protein complexed with hsp 90 is the same protein complexed with pp60 ${ }^{\mathrm{r}-\mathrm{sr}} / \mathrm{hsp} 90$ in cells transformed by the Rous sarcoma virus. J. Biol. Chem. 266 (1991) 16,436-16,440.

32. Dalman F. C., Sturzenbecker L. J., Levin A. A., Lucas D. A., Perdew G. H., Petkovitch M., Chambon P., Grippo J. F. and Pratt W. B.: The retinoic acid receptor belongs to a subclass of nuclear receptors that do not form "docking" complexes with hsp90. Biochemistry 30 (1991) 5605-5608.

33. Beckman R. P., Mizzen L. A. and Welch W. J.: Interaction of hsp70 with newly synthesized proteins: Implications for protein folding and assembly. Science, N.Y. 248 (1990) 850-854.

34. Skowyra D., Georgopoulos C. and Zylicz M.: The E. coli dna K gene product, the hsp70 homolog, can reactivate heat-inactivated RNA polymerase in an ATP hydrolysis-dependent manner. Cell 62 (1990) 939-944.

35. Rothman J. E.: Polypeptide chain binding proteins: catalysts of protein folding and related processes in cells. Cell 59 (1989) 591-601.

36. Howard K. J., Holley S. J., Yamamoto K. R. and Distelhorst C. W.: Mapping the hsp90 binding region of the glucocorticoid receptor. J. Biol. Chem. 265 (1990) 11928-11935.

37. Dalman F. C., Scherrer L. C., Taylor L. P., Akil H. and Pratt W. B.: Localization of the $90-\mathrm{kDa}$ heat shock protein-binding site within the hormone-binding domain of the glucocorticoid receptor by peptide competition. J. Biol. Chem. 266 (1991) 3482-3490.

38. Cadepond F., Schweizer-Groyer G., Segard-Maurel I., Jibard N., Hollenberg S. M., Giguere V., Evans R. M. and Baulieu E. E.: Heat shock protein 90 as a critical factor in maintaining glucocorticosteroid receptor in a nonfunctional state. J. Biol. Chem. 266 (1991) 5834-5841.

39. Carson-Jurica M. A., Lee A. T., Dobson A. W., Conneely O. M., Schrader W. T. and O'Malley B. W. Interaction of the chicken progesterone receptor with heat shock protein (HSP) 90. J. Steroid Biochem. 34 (1989) 1-9.

40. Rexin M., Bush W. and Gehring U.: Chemical cross-linking of heteromeric glucocorticoid receptors. Biochemistry 27 (1988) 5593-5601.

41. Aranyi P., Radanyi C., Renoir J. M., Devin J. and Baulieu E. E.: Covalent stabilization of the nontransformed chick oviduct cytosol progesterone receptor by chemical cross-linking. Biochemistry 27 (1988) $1330-1336$. 